УДК: 528.8:681.7

G.W. Kamerman

\title{
Recent development of 3D focal plane arrays
}

Для построения трехмерного (3D) изображения в лазерных локаторах обычно используется сканирование луча и последовательный во времени опрос по дальности. При этом для измерения дальности используется импульсная модуляция. Размер изображения и частота кадров лазерного локатора с построением трехмерного изображения зачастую ограничиваются частотой следования импульсов и эфффективностью сканирования. Повышение частоты следования импульсов может увеличить размер кадра, но только за счет неоднозначности отсчета по дальности и возрастания сложности сканирующего устройства и передатчика. В последнее время разработаны фокальные микроструктуры, включающие В себя схемы для измерения времени задержки сигнала. Эти устройства могут существенно упростить конструкцию лазерного локатора, увеличив при этом размер изображения и частоту кадров без внесения неоднозначности измерения дальности. Настоящая работа посвящена рассмотрению наиболее важных аспектов построения локаторов на базе интегрированных фокальных микроструктур.

Laser radars have traditionally employed beam scanning and sequential range interrogation in order to generate three-dimensional (3D) images. Pulsed modulation is commonly used in order to measure range. The image size and image rate of 3D or range imaging laser radars are frequently limited by the pulse repetition rate and by scanner efficiency. Increases in the pulse repetition may increase frame size and frame rate, but only at the expense of increased range ambiguity and increased complexity of the scanner and transmitter. Staring arrays that incorporate time of arrival measurements have recently become available. These arrays have the potential of simplifying instrument design while increasing image size and image rate without increasing range ambiguity. In our work, we discuss most sensitive aspects of ladar design using focal plane arrays.

Ключевые слова: лазерный локатор, измерение дальности, фоокальные микроструктуры, трехмерное изображение.

\section{Background}

Recent developments in staring arrays for three-dimensional imaging laser radars have attempted to overcome many of the limitations of "flying-spot" scanned laser radars and previous staring or "flash" illumination laser radars based upon commercial components. Consequently, it is useful to review these previous approaches. Each approach has its own engineering advantages and disadvantages.

One of the earliest attempts to implement 3D imaging, staring array laser radar employed a gain modulated CCD array and a sinusoidal, amplitude modulated (AM) transmitter. The gain of the CCD was modulated by placing it behind a microchannel plate, whose bias voltage was modulated at the AM transmitter frequency [1] The intensity incident on the CCD will be highest when the distance to the target is such that the received signal is in phase with the receiver. Likewise it will be the lowest when the range is such that the receiver signal is out of phase with the receiver modulation. The intensity falling on the CCD is now a measure of the range to the target and the target reflectivity. A second measurement with the receiver gain constant in order to eliminate target reflectivity effects. This technique is currently employed to conduct on-orbit inspection of the thermal protection tiles on the exterior of the Space Shuttle [2] after each launch (Fig. 1).

This architecture has several advantages. It permits large image frame size using only low cost, commercial components and without scanning. Detector size and pitch are relatively small $(\sim 10$ $\mu \mathrm{m})$ which enables good spatial resolution with modest focal length optics. Unfortunately, the dynamic range is limited and saturation corrupts the measurement. Furthermore, this technique is unable to resolve the range of multiple objects within the instantaneous field of view (IFOV) of the detector and the range measurement is ambiguous in the gain-modulated wavelength. High range precision requires high frequency modulation. Unfortunately, high frequency modulation also results in short ambiguity ranges. Multiple measurements at multiple, non-harmonic modulation frequencies are needed in order to resolve the AM range ambiguity. The need for multiple measurements in order to normalize reflectivity effects and to resolve range ambiguities 
reduces the effective frame rate to a fraction of the native CCD frame rate. Target motion, sensor motion or line-of-sight jitter during these multiple measurement can corrupt the final 3D image in unpredictable ways.

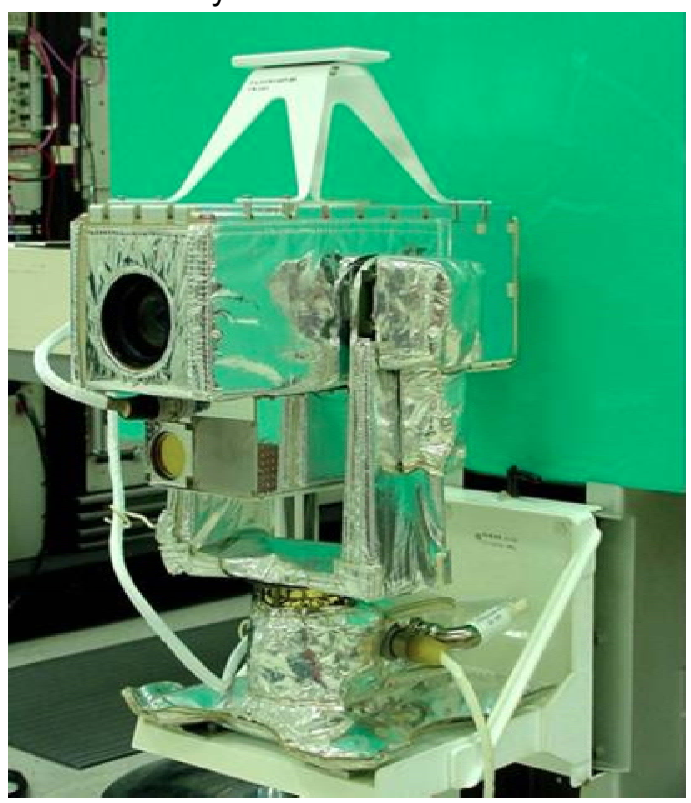

Fig. 1. Gain modulated staring laser radar developed by Sandia National Laboratory is used to examine the Space Shuttle Thermal Protection System on orbit

Another early approach used a pulsed transmitter and a streak tube receiver to determine the time of arrival. This approach did not suffer from the range ambiguity of the gain-modulated receiver. A fiber bundle was used to couple the received image to the streak tube and to re-format the twodimensional array into a line array. The line array was oriented with its axis perpendicular to the streak tube deflection axis. The position of the image of the target along the deflection axis is now a measure of the range.

The complexity of the fiber bundle limits the practical frame size. The largest known example of this technique is only $32 \times 32$ [3]. As a result, a variant was demonstrated which utilized multiple slit images on a single streak tube. In either technique, the width of the streak tube physically limits the span of ranges (i.e., the image range depth) that may be measured simultaneously. Reducing the sweep rate may increase this span, but this also produces a proportionate reduction in the range resolution and precision

Another tube based 3D imaging, staring laser radar receiver architecture used a photomultiplier (PMT) tube with a pixilated anode. This enabled single photon sensitivity and nearly linear response over a very wide dynamic range. The effective frame size was small, typically no larger than $10 \times 10$, and the detector pitch was quite large
( 3 $\mathrm{mm}$ ) [4] which required long focal length optics in order to achieve usable spatial resolution. In addition, discrete electronics were required for each anode element. This significantly increased the parts count, size, weight, and power consumption, but the sensor was still sufficiently small to be mounted and tested from a small Unmanned Air Vehicle (UAV) The entire system had a mass of less than 34 kilogram and collected 2.2 million measurements per second from an altitude of 1 kilometer. The use of discrete timing electronic also enabled higher precision timing measurements and, consequently, higher ranges precision [5]. Range precision was approximately 5 centimeters.

\section{Staring Detector Arrays}

One of the first detector arrays for developed specifically for laser radar employed a sinusoidal, AM transmitter where the AM frequency was chirp modulated [6]. The gain of the receiver was also modulated with this same FM/AM waveform. The received FM/AM signal is then further modulated by the FM/AM gain of the receiver and form a heterodyne signal. The frequency of the heterodyne signal is now proportionate to the range and the FM rate of the AM. This system demonstrated for the first time range resolved measurements using an AM optical waveform. Although effective, the system was not efficient. Sensitivity and, therefore, range was limited. Arrays of 32 by 32 detectors have been demonstrated. Field tests were limited to stationary, tripod-mounted tests.

One of the first attempts to implement more conventional radar processing in a staring laser radar used an array of avalanche photodiodes that were bump bonded to a matching array of Read Out Integrated Circuit (ROIC) timing electronics positioned directly behind the detectors [7]. The transmitter flood illuminated the field of view with a laser pulse. Since these detectors operated in a proportional mode and the timing electronics employed a form of centroid detection, the timing precision was not limited to the pulse length alone. Unfortunately, the size of the ROIC timing electronics forced the detector pitch to be quite large $(\sim 150 \mu \mathrm{m})$. Long local length optics was then required in order to achieve useful spatial resolution and lenslet arrays, place directly in front of the detectors are used to improve the effective fill factor of the array. In addition, the centoid detection electronics had a limited dynamic range and was sensitive to pulse broadening produced by distributed scatterers. Arrays as large as 128 by 128 are commercially available. Frame rate is limited to a few hundred frames per second (Fig. 2). 


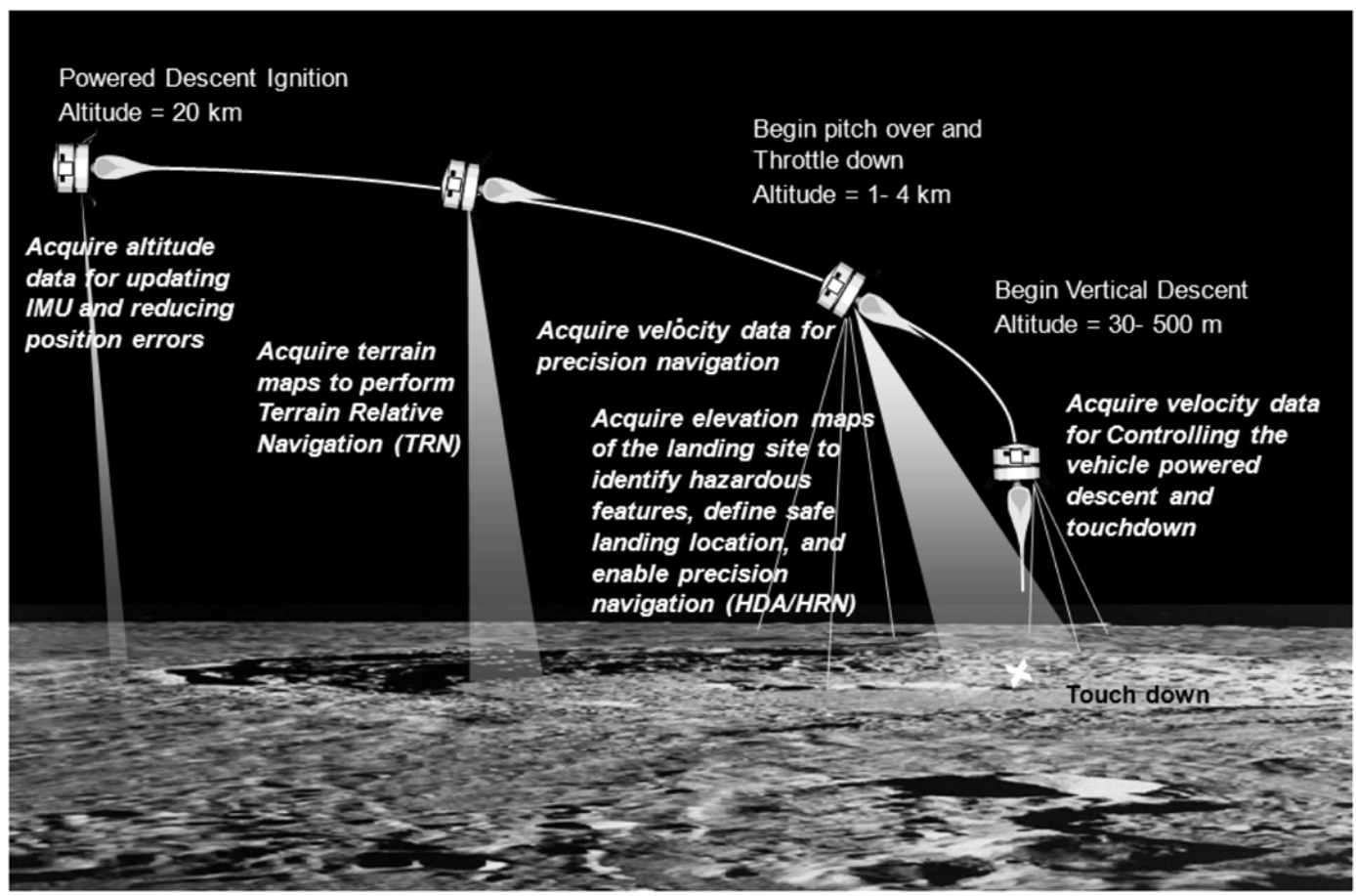

Fig. 2. Operational concept for 3D staring array ladar landing sensor

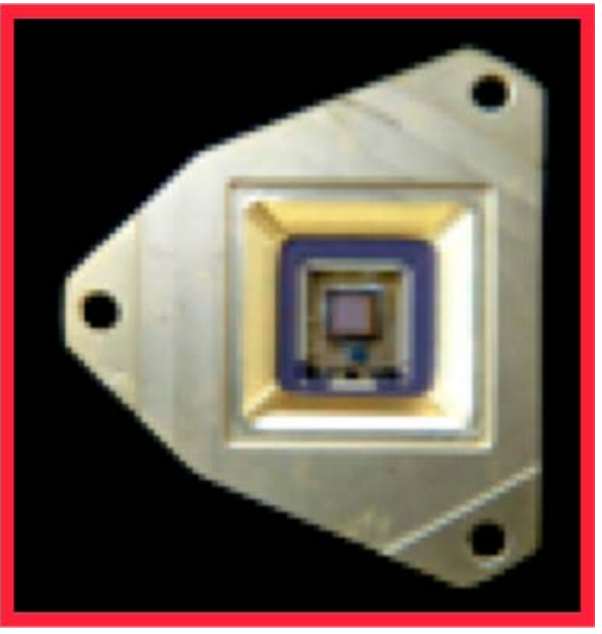

Fig. 3. 32 x 32 Geiger Mode APD Array developed by MIT Lincoln Laboratory is bump bonded to matching set or $2 \mathrm{GHz}$ timing counters

The latest generation of 3D detector arrays is a radical departure from previous approaches. These arrays employ APD arrays that are bump bonded to a purely digital, ROIC timing electronics [8]. However, unlike previous approaches, the detectors are biased above their breakdown voltage and operate in the Geiger mode. The detectors will continue to hold off the bias voltage for a short period of time (typically for a few microseconds). During this period of time, the absorption of a single photon can generate charge carriers and trigger the diode to breakdown. This transient then latches the value of the counter in the digital timing circuit. This results in single photon sensitivity but, since the response saturates with a single photon, an individual detection event cannot be distinguished from background or thermal noise. However, since signal photons from separate measurements are temporally and spatially correlated and spurious noise is not, the received signals may be distinguished from noise by multiple interrogations [9].

Like previous staring array detector arrays, the detector pitch is limited by the size of the ROIC and not the size of the detector (Fig. 3). Experimental arrays up to 64 by 256 detectors have been produced with a pixel pitch as small as $50 \mu \mathrm{m}$. However, all commercially available arrays have 32 by 32 elements on a $100 \mu \mathrm{m}$ pitch. Lenslet arrays are used to improve the fill factor. Dark count rate and quantum efficiency are both dependent upon over bias voltage. Our tests have shown that when the over bias voltage is set so that the dark count rate is approximately 2,000 counts per detector per second, that the input referenced quantum efficiency is generally $30 \%$ to $35 \%$. Frame rates are limited by the ROIC. The maximum rate depends upon the manufacturer but range from about $20 \mathrm{KHz}$ to over $200 \mathrm{kHz}$.

\section{Conclusions}

The current generation of staring array detector arrays has enabled laser radars with greater sensitivity and wider area coverage [10]. They are a significant advance over previous technologies, but still have several, serious limitations. The detector pitch is large, the quantum efficiency is lower than is desired and the format is still small. In addition, the response of these Geiger mode arrays saturates with a single photon. There is still a need for 
larger arrays of detector on a smaller pitch with wider dynamic range.

\section{References}

1. Garcia P., Anthes J.P., Pierce J.T., et al. Characterization of a scannerless ladar system. Proc. SPIE, 1993, Vol. 1936, p. 23-30.

2. Lamoreux J.C., Siekierski J.D., Carter J.P.N. Space Shuttle thermal protection system inspection by 3D imaging laser radar. Proc. SPIE, 2004, Vol. 5412, p. 273-281.

3. Redman B.C., Griffis A.J., Schibley E.B. Streak tube imaging Lidar (STIL) for 3D imaging of terrestrial targets, DTIC Report Number ADA 392466, 2000.

4. Degnan J.J. Photon-counting 3D imaging lidars. Sigma Space Corporation, Lanham, MD, Presentation to Laserfest, Arlington, VA, 27.03.2010.

5. Degnan J., Machan R., Leventhal E., et al. Inflight performance of a second-generation photon-counting 3D imaging lidar. Proc. SPIE, 2008, Vol. 6950, 695007.
6. Stann B., Redman B.C., Lawler W., et al. Chirped amplitude modulation ladar for range and Doppler measurements and 3D imaging. Proc. SPIE, 2007, Vol. 6550, 655005.

7. Stettner R., Bailey H., Silverman S. Threedimensional flash ladar focal planes and time dependent imaging. International Symposium on Spectral Sensing Research, 2006, Bar Harbor, Maine.

8. Albota M.A., Aull B.F., Fouche D.G., et al. Three-dimensional Imaging laser radars with Geiger-mode avalanche photodiode arrays. Lincoln Lab. Journal, 2002, Vol. 13, No. 2, p. 351-370.

9. Marino R.M., Davis W.R. Jigsaw: a foliagepenetrating $3 \mathrm{D}$ imaging laser radar system. Lincoln Lab. Journal, 2005, Vol. 15, No. 1, p. 23-36.

10. Sudharsanan R., Yuan P., Boisvert J., et al. Single photon counting Geiger mode In$\mathrm{GaAs}(\mathrm{P}) / \mathrm{InP}$ avalanche photodiode arrays for 3D imaging. Proc. SPIE, 2008, Vol. 6950, $69500 N$. 